University of Wollongong

Research Online

Faculty of Engineering and Information

Faculty of Engineering and Information

Sciences - Papers: Part A

Sciences

$1-1-2012$

\title{
Momentum balance method and estimation of boundary shear stress distribution
}

Shu-qing Yang

University of Wollongong, shuqing@uow.edu.au

Nadeesha Chamini Dharmasiri

University of Wollongong, ncdkl861@uowmail.edu.au

Yu Han

University of Wollongong, yh916@uowmail.edu.au

Follow this and additional works at: https://ro.uow.edu.au/eispapers

Part of the Engineering Commons, and the Science and Technology Studies Commons

Research Online is the open access institutional repository for the University of Wollongong. For further information contact the UOW Library: research-pubs@uow.edu.au 


\title{
Momentum balance method and estimation of boundary shear stress distribution
}

\begin{abstract}
Determination of local boundary shear stress is an important topic in hydraulic engineering. When attempting to determine this from a very thin boundary region, it is a difficult one because it requires special skills and instruments to treat the measured data. With the advent of new equipment, like the acoustic Doppler velocity profilers (ADVP), all the velocity profiles in the primary flow region can be simultaneously measured, and it is necessary to develop a new method to estimate the boundary shear stress using the primary flow data. This paper presents such a method called the momentum balance method (MBM). A theoretical relationship between the boundary shear stress and parameters of the primary flow region has been established. The results obtained from MBM agree reasonably well with other methods, indicating that the new method is workable in closed duct flows, and possibly its applicability can be extended to a wide range of boundary conditions.
\end{abstract}

\section{Keywords}

shear, momentum, stress, balance, disbribution, method, estimation, boundary

Disciplines

Engineering | Science and Technology Studies

\section{Publication Details}

Yang, S., Dharmasiri, N. Chamini. \& Han, Y. (2012). Momentum balance method and estimation of boundary shear stress distribution. Journal of Hydraulic Engineering, 138 (7), 657-660. 


\title{
Momentum Balance Method (MBM) and Estimation of Boundary Shear Stress Distribution
}

\author{
Shu-Qing Yang ${ }^{1}$, Nadeesha Dharmasiri ${ }^{2}$ and Yu Han $^{3 *}$
}

\begin{abstract}
Determination of local boundary shear stress is an important topic in hydraulic engineering. When attempting to determine this from a very thin boundary region, it is a difficult one because it requires special skills and instruments to treat the measured data. With the advent of new equipment, like the Acoustic Doppler Velocity Profilers (ADVP), all the velocity profiles in the main flow region can be simultaneously measured, and it is needed to develop a new method to estimate the boundary shear stress using the main flow data. This note presents such a method called Momentum Balance Method (MBM). A theoretical relationship between the boundary shear stress and parameters of main flow region has been established. The results obtained from MBM agree reasonably well with other methods, indicating that the new method is workable in closed duct flows and possibly its applicability can be extended to a wide range of boundary conditions.
\end{abstract}

Subject Headings: Boundary shear; Reynolds stress, Secondary flow; Turbulent flow; Velocity distribution, shear stress distribution.

\footnotetext{
${ }^{1}$ Assoc. Prof., School of Civil, Mining and Environmental Engineering, Univ. of Wollongong, NSW 2522, Australia.EMAIL:shuqing@uow.edu.au

${ }^{2} \mathrm{PhD}$ candidate, School of Civil, Mining \& Environmental Engineering, Univ. of Wollongong, NSW 2522, Australia.Email:ncdkl861@uow.edu.au.

${ }^{3}$ corresponding author, $\mathrm{PhD}$ candidate, School of Civil, Mining \& Environmental Engineering, Univ. of Wollongong, NSW 2522, Australia.E-mail:yh916@uow.edu.au,.
} 


\section{Introduction}

Estimation of the local or mean value of boundary shear stress is required in almost all hydraulic engineering problems, such as the computation of flow resistance, sidewall correction, sediment/pollutant transport or diffusion, dispersion, channel erosion or deposition, and designs of channels (e.g. Dey and Lambert 2005; Dey and Raikar 2007; Dwivedi et al. 2010). Substantial discrepancies in these predictions can occur due to the inaccurate estimation of boundary shear stress. The estimation of boundary shear stress has been studied extensively by many researchers, but it remains one of the most difficult parameters in hydraulics (Wilcock1996).

The near boundary region is the source of turbulent eddies. It is also the place where turbulent energy is dissipated into thermal energy due to resistance (Chien and Wan, 1999). As this region has the greatest velocity gradient and highest turbulence, it is also the most difficult layer from which to obtain reliable experimental data. For a bed covered by sediment particles, $\mathrm{Yu}$ and Tan (2006) found that a slight change in the velocity datum could lead to significant discrepancy in the boundary shear stress. To measure boundary shear stress, the Preston tube has been used to directly measure the turbulent skin friction (Knight and Patel 1985; Rhodes and Knight 1994). The hot-film technique has also been used for the detailed investigation of wall shear stress (Bruun, 1995). Recently, a hot-element probe was developed (Wietrzak and Lueptow, 1994), which can measure instantaneous fluctuations of the wall shear stress when the probe is placed very near to or in contact with the wall. More recently, wall-shear-stress measurements were carried out using liquid-crystal coating, which is thin enough to detect the local wall-shear-stress distribution (Redaet al. 1997). 
As opposed to the near boundary region, measurement in the main flow region is relatively simple and reliable, especially with the recent developments in instrumentation. Some new instruments can measure all the velocity profiles simultaneously; for example, the Acoustic Doppler Velocity Profiler (ADVP) can measure the 3-D velocity field from the water's surface to the bottom (Bagherimiyab and Lemmin, 2011). Like other instruments, ADVP has a very high noise level in the near boundary region, thus the dataset from the near boundary region are less reliable. Therefore, it is natural to ask whether the boundary shear stress can be estimated using the measured data in the main flow region.

The objective of this note is to develop a novel method whereby the boundary shear stressis determined using the main flow data rather than the viscous sub-layer measurements. As the first step towards this goal, only a simple rectangular duct flow is discussed in this study. This simple boundary geometry and non-existence of the free surface effect can simplify boundary shear stress calculation (Yang and Lim, 2005).

\section{Theoretical considerations}

Local boundary shear stress is an important fluid dynamic parameter. Unfortunately, it is often difficult to measure directly over movable beds and especially under field conditions, as all existing methods need to measure the flow structures in the near boundary region. Hence, a new method to avoid this shortcoming is needed.

For a steady, uniform, and fully developed turbulent flow as shown in Fig. 1, Yang(2010) established the momentum balance equation. Taking the element "OMPN" as an example, the gravitational force component for a unit length in the $x$-direction has to be balanced by the friction force on the interface of element "OMPN" : 
$\rho g A_{O M P N} S_{f}=\oint_{s} \tau d s=\int_{M}^{P} \tau_{1} d s+\int_{P}^{N} \tau_{2} d s+\int_{N}^{O} \tau_{3} d s+\int_{O}^{M} \tau_{4} d s$

where $\rho$ is density of fluid; $g$ is the gravitational acceleration; $A_{O M P N}$ is the area of flow region OMPN shown in Fig. 1; $S_{f}$ is the energy slope; $\tau$ is the shear stress on the interface of OMPN, $s$ denotes the length of the interface, $\tau_{1}$ to $\tau_{3}$ are shear stresses on the interface MP, NO and NP, respectively; $\tau_{4}$ is the boundary shear stress to be determined; $\rho g A_{O M P N} S_{f}$ is the gravitational force component in the streamwise direction. Yang (2009) showed that the shear stresson an interface should be expressed by:

$$
\tau_{x n}=\mu \frac{\partial u}{\partial \mathrm{n}}-\rho u v_{\mathrm{n}}-\rho \overline{u^{\prime} v_{\mathrm{n}}^{\prime}}
$$

where " $\mathbf{n}$ " represents the normal direction of the interface, $\rho u v_{\mathrm{n}}$ is the momentum flux caused by secondary currents, and $v_{\mathbf{n}}$ is the velocity component of secondary flow normal to the interface. In the Cartesian coordinate system, Eq. 2 can be rewritten in the following form:

$$
\begin{aligned}
& \tau_{x y}=\mu \frac{\partial u}{\partial y}-\rho u v-\rho \overline{u^{\prime} v^{\prime}} \\
& \tau_{x z}=\mu \frac{\partial u}{\partial z}-\rho u w-\rho \overline{u^{\prime} w^{\prime}}
\end{aligned}
$$

where $\tau_{x y}$ and $\tau_{x z}$ denote the shear stress on the horizontal and vertical interface, respectively; $u$ and $u^{\prime}, v$ and $v^{\prime}, w$ and $w^{\prime}$ are the mean and fluctuating velocities in $x, y$ and $z$ directions, respectively. In the main flow region, the viscosity effect can be ignored. 

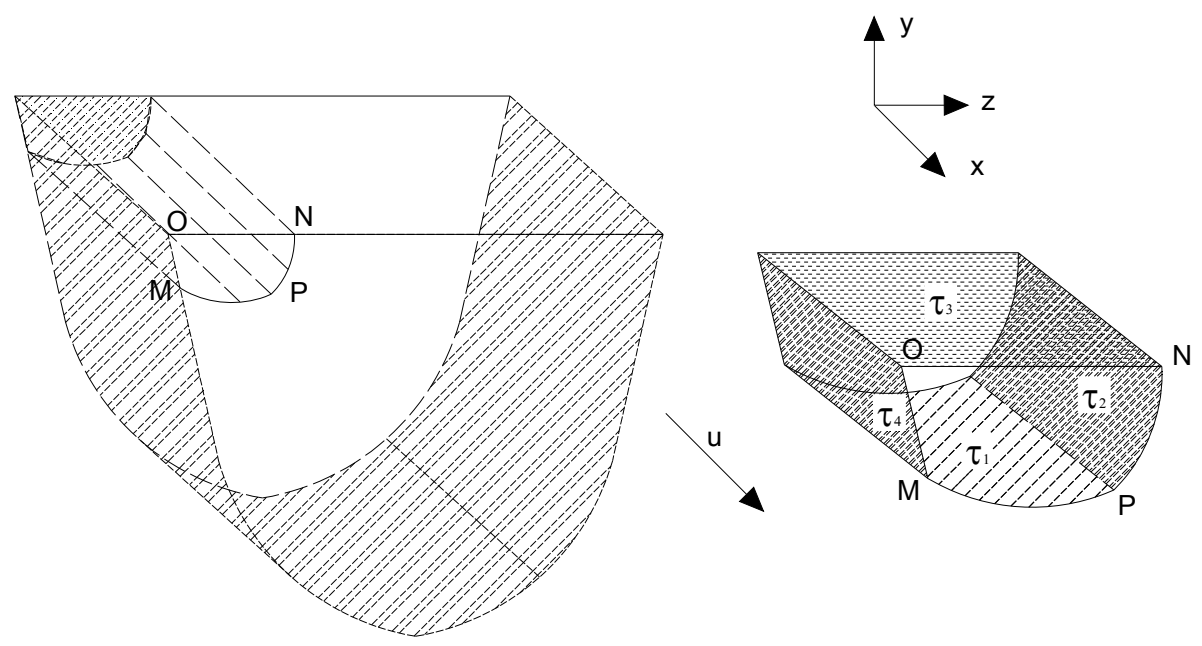

Fig. 1 The calculation grids for arbitrary cross-sectional channel and coordinate system.

Therefore, for a control volume shown in Fig. 2, the averaged sidewall shear stress $\bar{\tau}_{(y, y+\Delta y)}$ (acting on boundary from $\mathrm{y}$ to $\mathrm{y}+\Delta \mathrm{y}$ on the $\mathrm{y}$-axis) can be evaluated using the horizontal shadowed strip and Eq. 1 can be written in the following form:

$$
\rho g A S=\oint_{S} \tau d s=\int_{0}^{z} \tau_{x y 1} d z+\int_{0}^{z} \tau_{x y 2} d z+\int_{y}^{y+\Delta y} \tau_{x z} d y+\int_{y}^{y+\Delta y} \tau_{(y, y+\Delta y)} d y
$$

where $A$ is the shadowed area in Fig. $2 ; \tau_{x y 1}$ and $\tau_{x y 2}$ are the shear stresses on the upper and lower horizontal interfaces, respectively; $\tau_{x z}$ is the shear stress on the vertical interface from the point $(z, y)$ to the point $(z, y+\Delta y)$. As the first three terms can be calculated from the measured velocities, the sidewall averaged shear stress from $y$ to $y+\Delta y$, i.e., the fourth term of Eq. 4 can be determined using this momentum balance equation. Thus the wall shear stress 


$$
\tau_{x z}(y+\Delta y / 2) \approx \frac{\int_{y}^{y+\Delta y} \tau_{x z}(y, y+\Delta y) d y}{\Delta y}
$$

can be calculated.

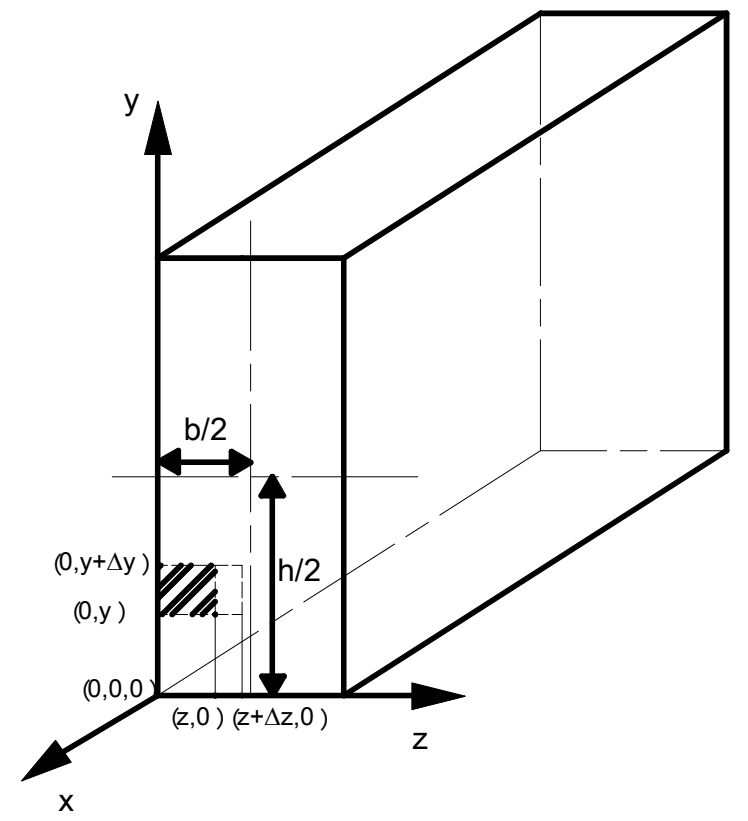

Fig.2 The closed duct flow used by Tracy (1965) and strips used for MBM calculation.

The numerator in Eq. 5 can be determined from the last term of Eq. 4 with the information from the main flow. Theoretically, the obtained sidewall shear stress should be the same if one changes the length of horizontal strip from $z$ to $z+\Delta z$, where $\Delta z$ is the horizontal space of measurement grids. But due to the existence of measurement errors, different sidewall shear stress could be obtained for different lengths. To avoid this, the averaged value is used when $\Delta z$ varies from zero to the central line in Fig. 2. Likewise, the bed shear stress can be evaluated from a vertical strip.

In contrast to Eq. (1), hydraulic engineers currently determine the boundary shear stress from the indirect measurement of friction velocity $u_{*}$ with different methods, such as: 
Log-law method (LLM): For a smooth boundary, the shear velocity $u_{*}$ along wall side can be determined from the measured mean velocity distribution $u(z)$ in conjunction with the logarithmic law.

$\frac{u}{u_{*}}=2.5 \ln \frac{u_{*} z}{v}+5.5$

where $v$ is kinematic viscosity. Eq. 6 is valid only in the inner region (Nezu and Nakagawa, 1993), even in the near wall region. Yang et al. (2005) questioned the validity of Eq. 6 as they found that the shear velocities on both sides of Eq. 6 are not identical. Reynolds shear stress method (RSM): $u_{*}$ can be determined from the measured Reynolds stress distribution (Nezu and Rodi 1986):

$\frac{\tau_{x y}}{\rho}=\overline{-u^{\prime} w^{\prime}}+v \frac{\partial u}{\partial z}=u_{*}^{2}\left(1-\frac{z}{h}\right)$

where $-\rho \overline{u^{\prime} w^{\prime}}$ is the Reynolds shear stress; $h$ is water depth. In near wall region $z \approx 0$, the measured Reynolds shear stress is constant, thus the boundary shear stress is the Reynolds shear in the constant shear stress layer.

Viscous sub-layer Method (VLM): It is well known that the velocity in the viscous sublayer $\left(u_{*} z / v<10\right)$ follows the linear distribution, i.e.,

$$
\frac{u}{u_{*}}=\frac{z u_{*}}{v}
$$

\section{MBM application to Smooth Rectangular Duct Flows}

To prove the MBM concept, Tracy's (1965) experimental data in a rectangular test duct is used. The experiment was conducted in a wind tunnel, air was supplied and the fan speed was controlled by varying the current to drive motor. The experimental cross section was 
5 inch $(0.127 \mathrm{~m})$ wide and 32 inch $(0.812 \mathrm{~m})$ high, the flow geometry and the coordinate system are shown in Fig.2. Hot-wire was used to measure the streamwise velocity $u$ and secondary currents $(v$ and $w)$. In the original paper the latter is discernible only for the region of $0<2 y / b<1$ and $0<2 z / b<1$, so we will mainly focus on this domain. The bed shear stress distribution on the side wall and the bottom are in unity in this domain. To compare MBM with other methods like LLM, VLM and RSM, the obtained sidewall and bed shear stresses are normalized by the mean sidewall and bed shear stress.Thus, in order to compute the bed shear stress, it is noted each velocity profiles represented the velocity $u$ varied with the vertical distance $y$ at a constant value of $z / h$, which can determine the $u_{*}$ results by Eq. 6 using the data of $y / h$ from 0.2 to 0.6 . Then, the $u_{*}$ values along lateral direction can be determined by LLM. Here, the velocity $u$ value closed to the bed from Tracy's(1965) measurements in the viscous sublayer layer $(y / h<0.02)$, would be used in calculation by VLM, i.e., Eq. 8 . As the distribution of $\overline{u^{\prime} v^{\prime}}$ relative to $u_{*}^{2}$ be showed in literature, it is used to investigate the boundary shear stress along the bed by RSM $(y / h<0.16)$. All the results are presented in Fig. 3 and Fig. 4 , respectively. Each bar represents the averaged local boundary shear stress over a strip.

Fig. 5 shows the comparison between the boundary shear stress obtained by MBM and other researchers' measured results, including Lawrence (1960), Leutheusser (1963) and Knight and Patel(1985). Only the data points with aspect ratio $(b / h)=1$ are included for comparison, these experiments were also conducted in wind tunnels, Preston tubes were used to determine the boundary shear stress. Experimental measurements for wall shear 
stress are made in a dimensionless form, i.e. $\tau_{b} / \overline{\tau_{b}}$ as well. It is seen that the relative bed shear stress $\tau_{b} / \overline{\tau_{b}}$ of MBM agrees reasonably well with these mentioned data.

In order to quantify the difference between these measured results and the obtained boundary shear stress by MBM, a standard deviation can be used $\left(\sigma=\sqrt{\sum_{i=1}^{N}\left(\tau_{i}-\overline{\tau_{i}}\right)^{2} / N}\right.$ here, $\tau_{i}$ refers to the calculated local boundary shear stress on same strips from different momentum balance elements), thus the standard deviations of each strip then can be calculated. The largest deviations as reported is $5.8 \%$, over the corner grid ( 0 to 0.05 and 0.05 to 0.1 ), it may be caused by the measurements of the velocity distribution, normally it is difficult to measure it accurately.

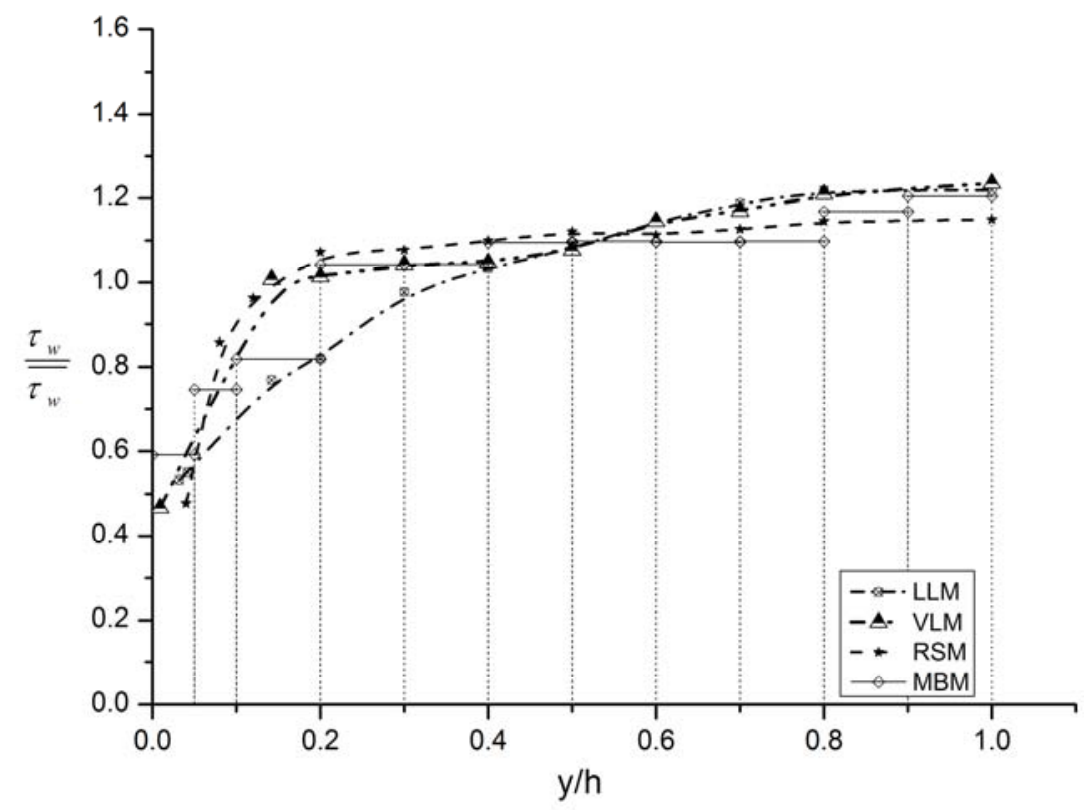

Fig. 3 Comparison of averaged sidewall shear stress distributions between MBM and other three methods based on Tracy's (1965) data. 


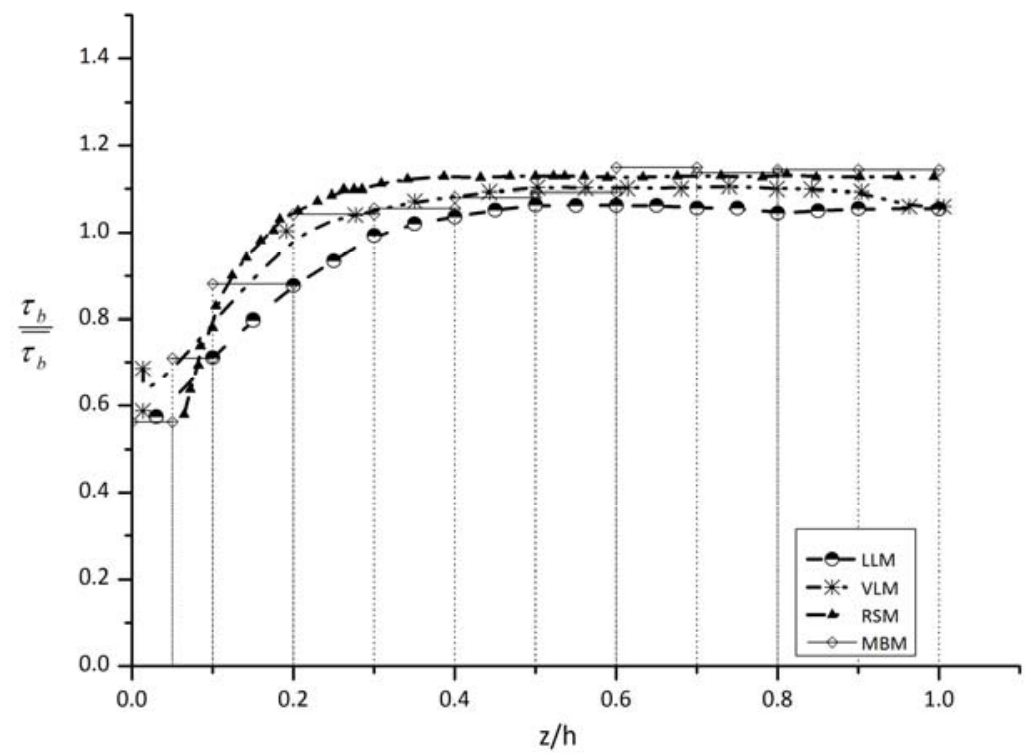

Fig. 4 Comparison of averaged bed shear stress distributions between MBM and other three methods based on Tracy's (1965) data

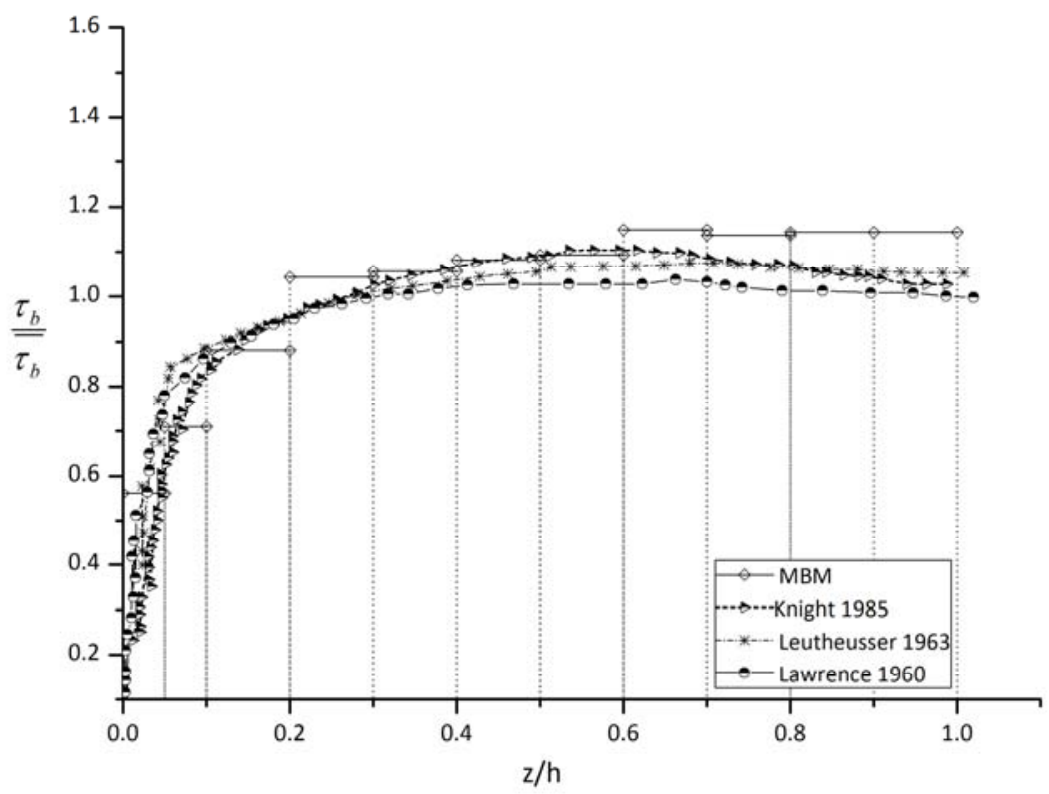

Fig.5 Comparison of averaged bed shear stress distribution between MBM for same aspect ratio

To validate the MBM, the relative error between MBM calculations (subscript $c$ ) and other methods (subscript $m$ ) has been defined as $E=\left|\tau_{c}-\tau_{m}\right| / \tau_{m}$. The sidewall shear stress shown in Fig 3 has the averaged $E$ of $2.5 \%, 1.92 \%$ and $3.3 \%$ between MBM and LLM, VLM, RSM respectively; the maximum value of $E(=15.5 \%)$ occurs in the strip of 
0.05-0.1. Similarly, Fig. 4 shows the comparison along the bed, the comparison shows that MBM works well and agrees with other methods (the averaged $E=-5.17 \%,-3.12 \%$ and $0.98 \%$ between MBM and LLM, VLM, RSM respectively), the maximum $E=13 \%$. The distribution of $\tau_{b} / \overline{\tau_{b}}$ is similar to the distribution of $\tau_{w} / \overline{\tau_{w}}$, so we only compared $\tau_{b} / \overline{\tau_{b}}$ with others' experimental data as presented in Fig. 5, where the averaged $E=-5.38 \%$ (Lawrence), $-5.51 \%$ (Leutheusser) and $-5.49 \%$ (Knight and Patel) when compared with MBM; and the maximum averaged $E=19 \%$ occurred near its centre line.

\section{Conclusions}

A new method (MBM) has been developed to estimate the boundary shear stress using main flow data based on the concept of momentum balance. Tracy's (1965) data and three existing methods were selected to test MBM. The results show that MBM is comparable with these existing methods and the following conclusions can be drawn from this study:

1) MBM can provide a reasonably good estimation for the distribution of boundary shear stress. No empirical coefficient or assumption is involved in the method. This method's reliability mainly depends on the accuracy of velocity measurement in the main flow region.

2) This study used smooth rectangular duct flows (Tracy1965) to test the MBM, and other existing methods were used for comparison including LLM, RSM and VLM. These comparisons showed that the MBM is comparable with these existing methods (averaged error $=2.5 \%, 1.92 \%$ and $3.3 \%$ between MBM and LLM, VLM, RSM respectively). The calculation of bed shear stress using MBM also agrees well with these alternative methods. When the estimated shear stress by MBM was compared 
with Lawrence (1960), Knight and Patel (1985) and Leutheusser (1963), the largest relative error was-5.51\%.

3) For smooth rectangular duct flows, MBM can assess the distribution of boundary shear stress. Its performance is as good as other methods. The applicability of MBM could be extended to complex boundary conditions, more research works should be carried out for its applicability in complex flows.

\section{References:}

Chien, N. N. andWan, Z. (1999). Mechanics of sediment transport, ASCE, Reston, VA. Dey, S., and Lambert, M. F. (2005). "Reynolds stress and bed shear in nonuniform unsteady open-channel flow.” J. Hydraul. Eng., 131(7), 610-614.

Dey, S., and Raikar, R. V. (2007). "Characteristics of Loose Rough Boundary Streams at Near-Threshold.” J. Hydraul. Eng., 133(3), 288-304.

Dwivedi, A., et al. (2010). "Hydrodynamic forces generated on a spherical sediment particle during entrainment.” J. Hydraul. Eng., 136(10), 756-769.

Bagherimiyab, F., and Lemmin, U. (2011). "Turbulence and fine sediment suspension in accelerating and decelerating open-channel flow." Proc., 34th IAHR World Congress, Brisbane, Australia, 3362-3369.

Bruun, H. H. (1996). Hot-wire anemometry: Principles and signal analysis, Oxford University Press, Oxford, 272-286.

Hoagland, L. C. (1960). "Fully developed turbulent flow in straight rectangular ductssecondary flow, its cause and effect on the primary flow." Ph.D. thesis, MIT, Dept. of Mechanical Engineering, Cambridge, MA. 
Knight, D. W., and Patel, H. S. (1985). "Boundary shear in smooth rectangular ducts." J. Hydraul. Eng., 111(1), 29-47.

Leutheusser, H. J. (1963). “Turbulent flow in rectangular ducts.” J. Hydr. Div., 89(3), 119.

Lim, S. Y., and Yang, S. Q. (2005). "Simplified model of tractive-force distribution in closed conduits.” J. Hydraul. Eng., 131(4), 322-329.

Nezu, I., and Nakagawa, H. Ed. (1993). Turbulence in open-channel flows, Balkema Publishers, Rotterdam, The Netherlands.

Nezu, I., and Rodi, W. (1986). “Open channel flow measurements with a laser doppler anemometer.” J. Hydraul. Eng., 112(5), 335-355.

Reda, D. C., et al. (1997). "New methodology for the measurement of surface shear stress vector distributions.” AIAA J., 35(4), 608-614.

Rhodes, D. G., and Knight, D. W. (1994). "Distribution of shear force on boundary of smooth rectangular duct.” J. Hydraul. Eng., 120(7), 787-807.

Tracy, H. J. (1965). “Turbulent flow in a three-dimensional channel.” J. Hydr. Div., 91(6), 9-35.

Wietrzak, A., and Lueptow, R. M. (1994). "Wall shear stress and velocity in a turbulent axisymmetrical boundary-layer.” J. Fluid Mech., 259, 191-218.

Wilcock, N. P. R. (1996). "Estimating local bed shear stress from velocity observations." Water Resour. Res., 32(11), 3361-3366.

Yang, S.Q., et al. (2005). "Investigation of near wall velocity in 3-D smooth channel flows.” J. Hydraul. Res., 43(2), 149-157.

Yang, S.Q. (2009). "Mechanism for initiating secondary currents in channel 
flows.” Can. J. Civ. Eng., 36(9), 1506-1516.

Yang, S.Q. (2010). "Depth-averaged shear stress and velocity in openchannel flows.” J. Hydraul. Eng., 136(11), 952-958.

Yu, G., and Tan, S.-K. (2006). "Errors in the bed shear stress as estimated from vertical velocity profile.” J. Irrig. Drain. Eng., 132(5), 490-497. 